\title{
INTERACTIVE COLLISION DETECTION FOR FREE-VIEWPOINT VIDEO
}

\author{
Bert De Decker, Tom Mertens, Philippe Bekaert \\ Expertise Centre for Digital Media, Hasselt University, Wetenschapspark 2, Diepenbeek, Belgium \\ bert.dedecker@uhasselt.be,tom.mertens@uhasselt.be,philippe.bekaert@uhasselt.be
}

Keywords: Video-based graphics, animation and rendering; collision detection; rigid body simulation; visual hull;

\begin{abstract}
We present a novel way of interacting with a virtual 3D scene in the context of free-viewpoint video. Using a multi-camera setup, our technique detects collisions between virtual objects and real objects, including people. We perform collision computations directly on the image data, as opposed to reconstructing the full geometry of the subject. This reduces implementation complexity, and moreover, yields interactive performance. We demonstrate the effectiveness of our technique by incorporating it in a rigid body simulation. The subject can interact with virtual objects and observe his or her actions while being able to adjust the viewpoint, all in real-time.
\end{abstract}

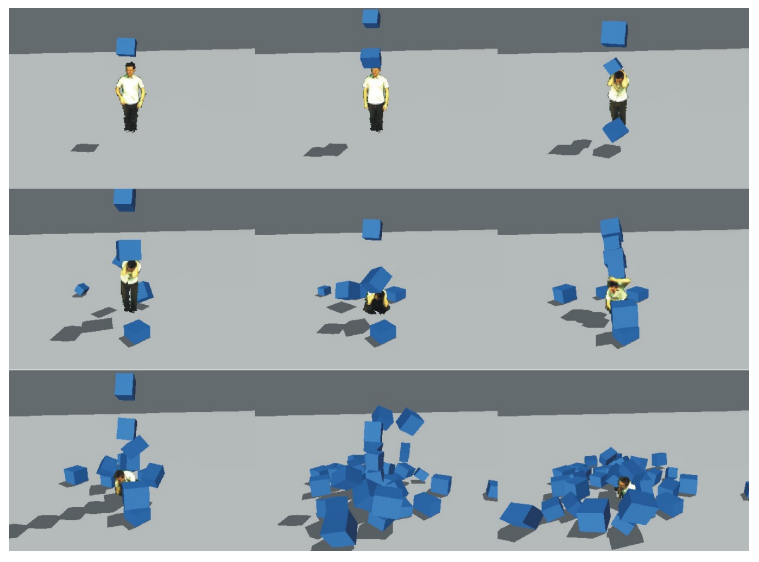

Figure 1: A person, captured by multiple calibrated digital video cameras, interacts with a rigid body simulation at interactive speeds.

\section{INTRODUCTION}

A lot of work has been invested in reconstructing 3D shapes from still or moving images in the last decades in the field of Computer Vision, and recently also in Computer Graphics. In this context, free-viewpoint video has emerged as a extension of traditional live-action video, which enables a more immersive experience for the observer. In particular, the observer is able to view the scene from any viewpoint as opposed to a single viewpoint. Scenes one typically considers involve some sort of human performance, possibly including other moving elements. To implement such a system, a multi-camera setup is used to reconstruct shapes and material properties of the scene, which can then be used to render novel views. This process typically consists of three steps: capturing the scene and/or performance, analysis of video frames and synthesis of novel viewpoints. Analysis and synthesis might be performed offline, or offline and online, respectively. Alternatively, the entire process might be implemented as a real-time system such that live performances can be viewed in realtime, akin to a live television broadcast. Since we are dealing with interaction (usually involving a human subject), our work is mostly applicable to the latter application domain.

In this paper, we go beyond free viewpoint video by adding interactions between the real world and a virtual scene. More precisely, we introduce a technique to determine collisions between real and virtual objects. A straightforward approach to tackle this problem, would be to apply 3D reconstruction technique " $\mathrm{X}$ " to obtain a triangle mesh of the scene, on 
which standard collision detection schemes can be applied. However, it would be better to avoid this intermediate reconstruction step, for the sake of simplicity and computational efficiency. Our technique is therefore designed to work directly on the information in the video frames.

In the classic collision detection problem, contact is determined from a full 3D description of the participating shapes (i.e. a triangle mesh). In our case, each camera only provides a discrete, $2 \mathrm{D}$ description of the real-world shapes. First of all, the information of the set of 2D frames acquired from each camera has to be aggregated to derive a collision test in 3 dimensions. We implement this based on the concept of a visual hull, i.e. the volume extruded from the silhouette of an object or subject. This information is readily available as a result of fast and simple foreground segmentation, and thereby avoids a costly 3D reconstruction step. Since camera registration is inherently a discrete process (granularity $=$ one pixel), we have to be careful in avoiding aliasing artifacts when determining the exact collision location. Finally, the analysis has to process data at a high bandwidth, since frames from multiple cameras have to be processed at each instant, while maintaining interactive performance. We therefore introduce a simple acceleration scheme to efficiently test the visual hull data. These issues will be explored in the remainder of the paper. We conclude with results and directions for future work.

\section{RELATED WORK}

Free Viewpoint Video Our collision detection technique was developed specifically with free viewpoint video in mind. Since our technique is fairly simple, and works directly on the video frame data, we are free to choose any existing free viewpoint video reconstruction and rendering technique, such as the voxel-based visual hull calculation by Hasenfratz et al. (Hasenfratz et al., 2003) or the real-time polygonal visual hull calculation by Matusik et al. (Matusik et al., 2001). For our experiments, we implemented the photohull calculation technique by $\mathrm{Li}$ et al. ( $\mathrm{Li}$ et al., 2004).

\section{Collision Detection between Virtual Objects} Many techniques have been developed for collision detection between only virtual objects (Guendelman et al., 2003; Baraff, 1992; Pauly et al., 2004; Heidelberger et al., 2004), cloth (Bridson et al., 2002; Govindaraju et al., 2005), deformable objects (Debunne et al., 2001; Teschner et al., 2005; Dewaele and Cani, 2004) , articulated objects (Redon et al., 2004) and fluids (Losasso et al., 2006) .

Interaction Techniques The technique presented here can also be seen as a way to interact with a virtual environment (Hand, 1997; Bowman and Hodges, 1997; Grossman et al., 2004). Yoshifumi et al. (Kitamura et al., 2003) implement interaction between real and virtual objects by defining a constrained set of physical laws. Xiyong et al. (Wang et al., 2005) present a system where users can manipulate scanned, articulated virtual representations of real objects.

Force Feedback In our work, we perform a one way interaction: the real world can interact with the virtual world, but not vice versa. There are also some techniques that provide interaction in 2 directions. An example of such a technique is presented by Lindeman et al. (Lindeman et al., 2004). They describe a system that gives haptic feedback to a user that walks around in a virtual environment. Since we allow arbitrary real-world objects in our scene, it is not readily possible to implement such feedback.

Collision Detection between Real and Virtual Objects Allard et al. (Allard and Raffin, 2006; Allard et al., 2006) present a physically-based animation system in which users can interact with the objects in the scene using a visual hull. They calculate a mesh representation of the visual hull of the real world and use this mesh to calculate the collision detection and response information. Breen et al. (Breen et al., 1996) and Hasenfratz et al. (Hasenfratz et al., 2004) also describe a system where some geometry of the real world is calculated as a preprocessing step in the collision detection between real and virtual objects. Stam (Stam, 2000) presents a method where a depth map is calculated by filming a person with a special camera, using this depth map a person can interact with a fluid simulation.

We bypass the mesh generation and work directly on the images which results in higher performance. Most related to our work is the system by Lok et al. (Lok et al., 2003). Instead of using an intermediate mesh representation of the real world, they rasterize every virtual triangle to all the input cameras to determine collisions using graphics hardware. However, they assume there is at most one collision between one virtual object and the visual hull of the real object at a time. This is true in some cases (for example a virtual ball bouncing off a wall). However, when a virtual box lies on a real table, for instance, this assumption breaks down. The main advantage of 
our algorithm over theirs, is the fact that we take into account multiple simultaneous collisions.

\section{OVERVIEW}

The main contribution of this paper is an algorithm to detect and respond to collisions between real and virtual objects. Collisions are determined from captured video data of real scene with multiple digital cameras.

The collision algorithm is based on the visual hull representation (Laurentini, 1994). When a virtual object intersects the visual hull of the real scene at a given time, the system will resolve this collision by moving the virtual object out of the visual hull and by flipping its velocity vector over the tangent plane of the visual hull at the collision point. This way, the virtual object is no longer intersecting the visual hull and is moving away from it after the collision. An important difference between traditional collision detection and this case, is that the shape of the real objects is only available at discrete time steps. So it is impossible to find to exact moment of contact. Instead of preventing collisions, the system will recover from them by computing an appropriate collision response vector that undoes the penetration, as introduced by Lok et al. (Lok et al., 2003). The distance the virtual object is moved to undo the penetration should be as small as possible, so the collision response vector is chosen to be perpendicular to the penetrated real-world object and its length is chosen to be such that the virtual object will no longer be in a state of collision in the next time step. Other definitions of the collision response vector could be used, but we found that this one gives good results while still allowing interactive speeds. For example a better collision response vector could be constructed by taking the speeds of the colliding objects into account. But since it is very hard to calculate the speed of the surface of the visual hull of the real object at interactive speeds, we can not use it for our purpose. In figure 2 a simple example is shown that illustrates the technique.

\section{POINT COLLISION DETECTION}

Before detailing our general collision detection scheme, we first focus on the special case of determining collision between a real object and a point. In the following section, this procedure will be generalized to arbitrary objects.

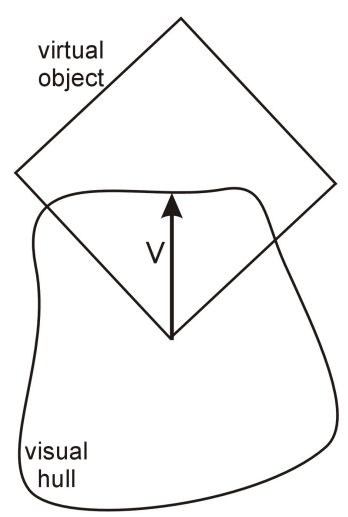

Figure 2: A simple example where a virtual object is intersecting the visual hull. The collision response vector is represented by $v$, the vector that is perpendicular to the visual hull. If the virtual object is translated along $v$, the virtual object is no longer intersecting the visual hull.

\subsection{Inside/Outside Test}

For every input camera, we compute the object's silhouette using simple background subtraction (Cheung et al., 2000), which forms the visual hull. Since we know the intrinsic and extrinsic parameters of each camera, each point can be projected into its view. By simply looking up the foreground/background classification and the corresponding pixel, we can determine whether the point is inside the hull.

Even though this test is extremely simple, when many such queries are executed, computational performance becomes limited by the sheer amount of memory access. Bounding volumes of the visual hull can be used to reduce the number of times an explicit image-based inside/outside test needs to be performed. We use 2 types of bounding volumes: the bounding frustum of the visual hull, and the axis aligned bounding box of this frustum. The bounding frustum is constructed from the center of projection of the corresponding camera and the $2 \mathrm{D}$ bounding rectangle of the actual visual hull. This rectangle can be obtained efficiently in a single pass by iterating over all visual hull pixels and keeping track of the extreme coordinate values. Before testing a point against the 4 planes that form the frustum, we first test it against the axis-aligned bounding box of the bounding frustum, as this test is even simpler.

\subsection{Collision Response}

If a point lies inside the visual hull, the collision response vector has to be calculated. Figure 3 shows an example where the collision response for point $a$ is 


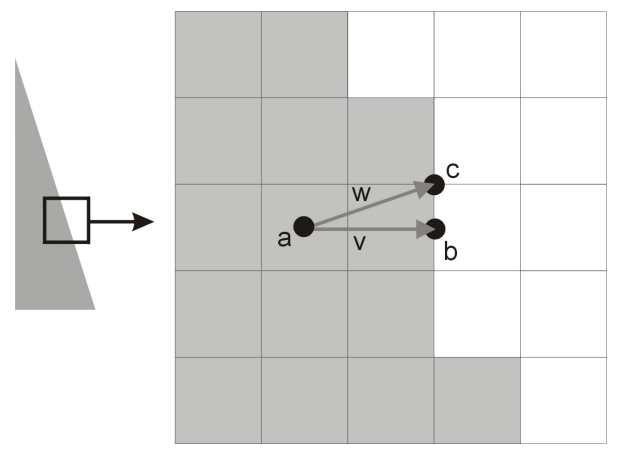

Figure 3: Left: one of the silhouette images. Right: a 5x5 window of this silhouette image, point a is intersecting the visual hull, point $b$ is the closest point on the surface, but with the wrong normal, $\mathrm{c}$ is the point on the surface with the right normal

needed.

As described in section 3, the collision response vector has to be perpendicular to the visual hull surface and when the rigid body is translated along this vector, it is no longer intersecting the visual hull.

The naive way to find this vector would be to find the point on the visual hull surface that is the closest to $a$ (point $b$ in the figure) and defining the collision response vector as the vector connecting $a$ and $b$ (vector $v$ is the figure). To find the point $b, a$ is projected to all the input images. For every iteration of the search algorithm, a square window around the projection of $a$ is considered for every input image. If one of the pixels of the window is not inside the silhouette image, than the projection of $b$ is found. To obtain $b$ we simply need to backproject its projection into 3D space. The initial size of the window is 3 by 3 pixels and it grows at every step of the iteration until we find the projection of $b$. But one pixel distance in one camera image corresponds to a different distance in 3D than one pixel distance in another camera image. The search window of cameras that are almost perpendicular to the surface grows faster than that of cameras that are at an angle with respect to the surface. The grow speed of the windows is calculated as follows: for every camera the pixel below and the pixel on the right of the pixel where $b$ was projected are projected back in 3D and the smallest distance between $b$ and these backprojected pixels is called $d$. So each camera has its own $d$ and the maximum of these values is called dmax. The growspeed for the window of a camera is now defined as $d \max / d$.

When point $a$ lies far away from the surface of the visual hull, the algorithm for the calculation of the collision response described above would be a good option and the direction of $v$ would be close to the surface normal. But due to the discrete nature of the input images, this presents a problem for points close to the surface of the visual hull. If $a$ was for example a boundary pixel, the length of $v$ would be half a pixel and there would only be 8 possible directions for $v$ : up, down, left, right, front and back. [When using $v$ to calculate the normals of the visual hull, the visual hull surface will not be smooth, instead there would be a sharp edge at points in space that are projected onto the edge of two neightbouring pixels that are part of the silhouette boundary of one of the input images.] We don't want the normal at one point of the surface of the visual hull because it is prone to the discrete nature of the images, instead we want the normal of some area around the point, integrating out the discrete nature of the images.

To construct a collision response vector with the same direction as this better surface normal, the following algorithm is used. The point $b$ is calculated as described above. Typically, there is only one input image for which the projection of $b$ lies on the silhouette boundary, for all the other images it will lie inside the silhouette. So only one input image provides information about the surface normal at $b$. The exception to this is when $b$ lies on an edge or on a corner of the visual hull, in that case the projection of $b$ lies on the silhouette boundary of multiple images. When this special case occurs, the first image for which the projection of $b$ lies on the silhouette boundary is chosen, the others are ignored and the algorithm acts as if the special case does not exist. So when the special case occurs, the algorithm doesn't return the normal at $b$, but the normal of another point that lies at the distance of one pixel from $b$. While this is not completely correct, this will not be visible in the final simulation and is a lot faster to calculate. So only one image is needed to calculate the 3D surface normal of the visual hull at point $b$. A 2D silhouette normal is calculated at the projection of $b$ in this image. For every pixel that is outside the silhouette and inside a $5 \times 5$ window around the projection of $b$, a $2 \mathrm{D}$ vector is created from the projection of $b$ to this pixel. The 2D silhouette normal is the average of all these vectors. The 3D normal vector is obtained by backprojecting the 2D silhouette normal to 3D space. The final collision response vector $w$ is defined as the vector connecting the collision point $a$ and point $c$ where $c$ is the intersection between the visual hull surface and the ray starting in $a$ in the direction of the previously calculated surface normal. The algorithm used to find $c$ is similar to the one used to calculate $b$, except that in this case no search window is used but a search line that grows longer and longer each iteration. 


\section{GENERAL COLLISION DETECTION}

To perform general collision detections with objects instead of points, we represent each object as a collection of points using surface sampling. For each individual point of an object, we perform the collision test and response computation as described in the previous section. We compute a single vector $V$ by averaging over all of the per-point response vectors of the object. For each sampled point, we search along this direction until a point on the hull boundary is found and a vector connecting this point and the sampled point is constructed. The response vector of the rigid body is obtained by taking the longest of these vectors. See figure 4 for an illustration.

The difference with the technique by Lok et. al. (Lok et al., 2003) is, they only consider one point of the surface of the virtual object during the calculation of the collision response. This is the reason why they can not model certain effects, for example a virtual box lying in rest on top of a real one. The technique presented here does not have this limitation because multiple points on the surface of the virtual object that are in collision with the visual hull are taken into account.

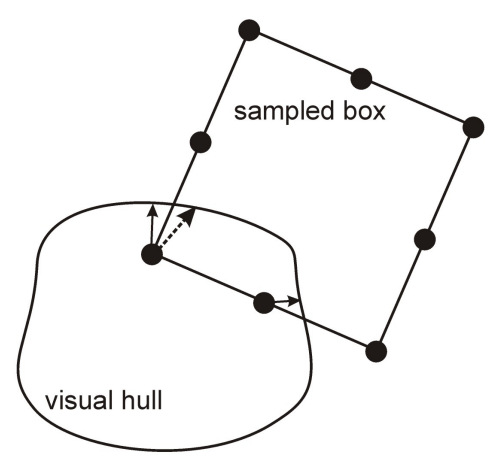

Figure 4: Point-based collision detection for arbitrary objects. For each point, we compute a collision response vector. By rescaling the average of these vectors, the response vector of the rigid body is obtained.

Bounding box tests can be used to cull entire objects for collisions tests: if the axis aligned bounding box of the visual hull does not intersect with axis aligned bounding box of the rigid body then the rigid body will not intersect with the visual hull either and further tests are not necessary.

If the virtual object is sampled too sparsely, it is possible that the detection "misses" some collisions. Because calculating the per-point test is very cheap, it is possible to sample the objects dense enough while still being able to perform the collision detection at high speeds. In our implementation the amount of samples used for a certain object depends on the size of the object. In the experiment shown in figure 1 between 250 and 500 samples per box are used. Another advantage is that all objects that can be point sampled can be dealt with, such as spheres, meshes and implicit surfaces. The only requirement on the pointsampling is that two neighbouring samples should lie close enough to each other in order that no part of the visual hull could pass between them. Uniform sampling will give the best accuracy for a given number of samples, but is no requirement.

\section{RESULTS}

\subsection{Collision Detection System}

The algorithm described above was implemented in a real-time free viewpoint video system. The camera setup consists of 7 cameras positioned in a hemisphere, and all the cameras are directed to the subject. The room is covered with green cloth to simplify the background subtraction. The cameras are calibrated using Svoboda's (Svoboda et al., 2002) calibration tool. The cameras are distributed across 3 PCs. These PCs are responsible for acquiring the images from the cameras and for calculating the silhouette images. The images along with the silhouette images are gathered on a central PC which performs the rigid body simulation, the collision detection and the visualization. The real-world scene is rendered by the Photohull plane sweeping algorithm by $\mathrm{Li}$ et al.(Li et al., 2004) and runs entirely on the GPU. We coupled our collision detection system with a rigid body simulator (Open Dynamics Engine(ODE, 2006)).

Our proof-of-concept implementation runs at approximately 10 frames per second when 7 cameras are used and the scene is rendered at a resolution of $640 x 480$. The system can calculate collision detection response information at about $500 \mathrm{~K}$ points per second. The bottleneck is not the central PC which does the visualization and collision detection, but the transmition of the images over the network. For the moment the images are not compressed when they are sent over the network, taking more time than strictly necessary. Simple run-length encoding of the visual hull images will likely improve performance significantly.

\subsection{Experiments}

In Figure 1, 5 and 6 we show some examples. 
In our first experiment, shown in Figure 5, a stack of virtual boxes on top of a real box is tipped over by lifting the real box. This demonstrates how our technique is capable of modeling virtual objects that are lying in rest on top of real ones, because we can deal with multiple simultaneous collisions. Figure 6 shows the interaction between a human and a pile of boxes. In the last experiment (Figure 1) we demonstrate a scene involving many virtual objects.

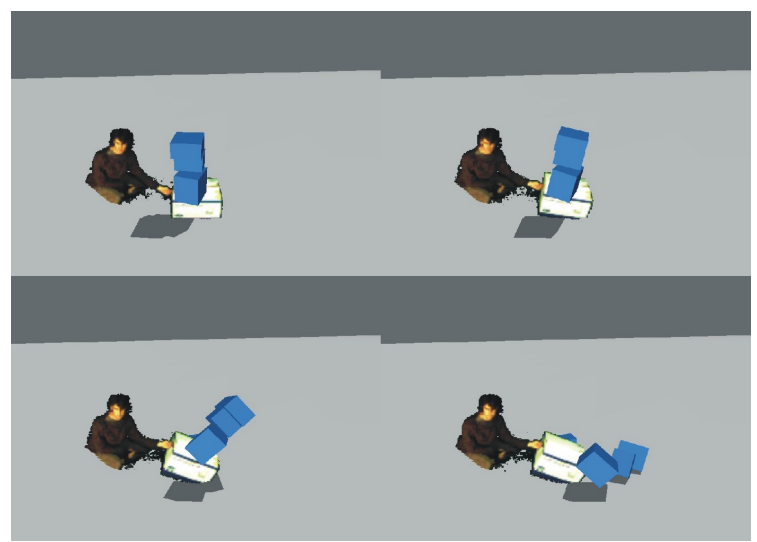

Figure 5: A stack of virtual boxes on top of a real box is tipped over by lifting the real box. This demonstrates how our technique is capable of modeling virtual objects that are lying in rest on top of real ones, due to correct treatment of multiple simultaneous collisions.

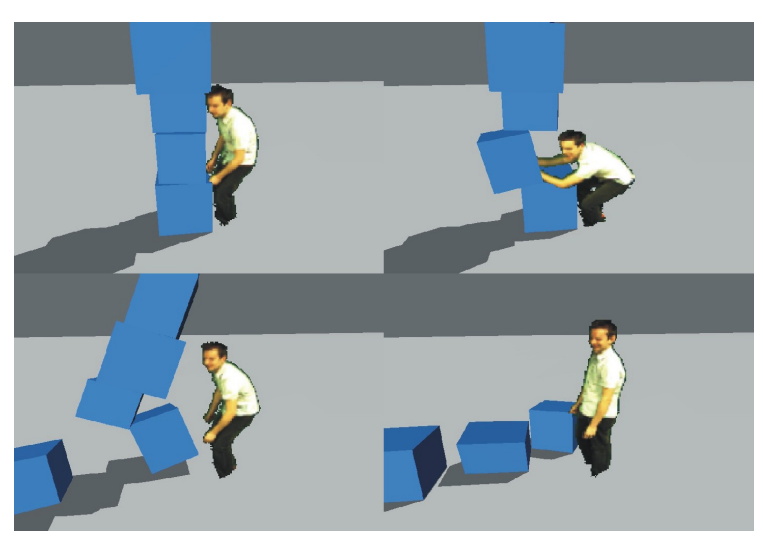

Figure 6: A real person topples a stack of virtual boxes.

\section{CONCLUSION AND FUTURE WORK}

We presented a novel technique that interactively handles collision detection between real and virtual objects in the context of a free-viewpoint video setup.
Using the concept of the visual hull extracted from a number of different views of the real-world scene, collisions are determined by point sampling the virtual objects, and performing simple and efficient inside/outside tests on these points. Our technique is fast enough for interactive applications, and compared to previous visual hull-based approaches, we are able to handle multiple simultaneous collisions.

At the moment, the system can not handle very fast moving objects. If at one instant, the visual hull is in front of a rigid body and at the next frame it is behind the rigid body, the contact that should have been detected is missed. To solve this, we need to know for every camera which pixels were occupied by the real object between the two time steps. We should also be able to query the visual hull in between two frames, so we can calculate the precise time of the collision of 2 fast moving objects. When objects are moving relatively slow as in our examples, we don't need this much precision to generate a plausible simulation.

The system could also be extended to make it possible for the users to grab virtual objects and move them around in the real world. One could for example have the user carry a button in his hand and when it is pushed, the visual hull would becomes sticky and objects touched by the user would stick to his body until he releases the button.

The algorithm is also very suited for parallel implementation on a cluster. Since we need multiple machines to connect the cameras anyway, we might as well use them to help in the collision calculations. The main advantage would not be the increased frame rate, but the possibility to use high resolution images. The speed of the algorithm presented here is independent of the resolution of the input images (except for the background subtraction), but it requires that all the images are gathered on one central machine which implies bandwidth limitations. When using a distributed approach, none of the images need to be send over the network, and all the calculations regarding one image are performed locally on the PC that gathered the image from the camera.

\section{ACKNOWLEDGEMENTS}

The authors acknowledge financial support on a structural basis from the European fund for regional development (ERDF), the Flemish institute for broadband communication (IBBT), as well as impuls financing from the transnationale Universiteit Limburg. 


\section{REFERENCES}

Allard, J., Franco, J., Menier, C., Boyer, E., and Raffin, B. (2006). The grimage platform: A mixed reality environment for interactions. In $I C V S$.

Allard, J. and Raffin, B. (2006). Distributed physical based simulations for large vr applications. In IEEE Virtual Reality Conference.

Baraff, D. (1992). Dynamic simulation of non-penetrating rigid body simulation. $\mathrm{PhD}$ thesis.

Bowman, D. and Hodges, L. (1997). Techniques for grabbing and manipulating remote objects in immersive virtual environments. In ACM Symposium on Interactive 3-D Graphics.

Breen, D., Whitaker, R., and Tuceryan, M. (1996). Interactive occlusion and automatic object placement for augmented reality. In Computer Graphics Forum, Blackwell Publishers

Bridson, R., Fedkiw, R., , and Anderson, J. (2002). Robust treatment of collisions, contact and friction for cloth animation. In conference on Computer graphics and interactive techniques.

Cheung, K. M., Kanade, T., Bouguet, J.-Y., and Holler, M. (2000). A real time system for robust 3d voxel reconstruction of human motions. In Proceedings of the 2000 IEEE Conference on Computer Vision and Pattern Recognition (CVPR 'OO), volume 2, pages 714 720 .

Debunne, G., Desbrun, M., Cani, M.-P., and Barr, A. H. (2001). Dynamic realtime deformations using space and time adaptive sampling. In ACM SIGGRAPH.

Dewaele, G. and Cani, M.-P. (2004). Interactive global and local deformations for virtual clay. In Graphical Models.

Govindaraju, N., Knott, D., Jain, N., Kabul, I., Tamstorf, R., Gayle, R., Lin, M., and Manocha, D. (2005). Interactive collision detection between deformable models using chromatic decomposition. In ACM SIGGRAPH.

Grossman, T., Wigdor, D., and Balakrishnan, R. (2004). Multi-finger gestural interaction with $3 \mathrm{~d}$ volumetric displays. In UIST '04: Proceedings of the 17th annual ACM symposium on User interface software and technology, pages 61-70, New York, NY, USA. ACM Press.

Guendelman, E., Bridson, R., , and Fedkiw, R. (2003). Nonconvex rigid bodies with stacking. In $A C M$ SIGGRAPH.

Hand, C. (1997). A survey of 3-d interaction techniques. In Computer Graphics Forum, Blackwell Publishers.

Hasenfratz, J., Lapierre, M., Gascuel, J., and Boyer, E. (2003). Real-time capture, reconstruction and insertion into virtual world of human actors. In Vision, Video and Graphics Conference.

Hasenfratz, J., Lapierre, M., and Sillion, F. (2004). A real-time system for full body interaction with virtual worlds. In Eurographics Symposium on Virtual Environments.
Heidelberger, B., Teschner, M., and Gross, M. (2004). Detection of collisions and self-collisions using imagespace techniques. In WSCG.

Kitamura, Y., Ogata, S., and Kishino, F. (2003). A manipulation environment of virtual and real objects using a magnetic metaphor. In ACM Symposium on Virtual Reality Software and Technology.

Laurentini, A. (1994). The visual hull concept for silhouette based image understanding. In IEEE PAMI.

Li, M., Magnor, M., and Seidel, H. (2004). Hardwareaccelerated rendering of photo hulls. In Computer Graphics Forum.

Lindeman, R. W., Page, R., Yanagida, Y., and Sibert, J. L. (2004). Towards full-body haptic feedback: The design and deployment of a spatialized vibrotactile feedback system. In ACM Virtual Reality Software and Technology (VRST).

Lok, B., Naik, S., Whitton, M., and Jr, F. B. (2003). Incorporating dynamic real objects into immersive virtua environments. In Proceedings of the 2003 symposium on Interactive $3 D$ graphics.

Losasso, F., Shinar, T., Selle, A., and Fedkiw, R. (2006). Multiple interacting liquids. In ACM SIGGRAPH.

Matusik, W., Buehler, C., and McMillan, L. (2001). Polyhedral visual hulls for real-time rendering. In Eurographics Workshop on Rendering.

ODE (2006). Open dynamics engine (ode).

Pauly, M., Pai, D. K., and Guibas, L. J. (2004). Quasi-rigid objects in contact. In Eurographics/ACM SIGGRAPH Symposium on Computer Animation.

Redon, S., Kim, Y. J., Lin, M. C., and Manocha, D. (2004). Fast continuous collision detection for articulated models. In ACM Symposium on Solid Modeling and Applications.

Stam, J. (2000). Interacting with smoke and fire in real time. In Communications of the ACM.

Svoboda, T., Hug, H., and Gool, L. V. (2002). Viroom - low cost synchronised multicamera system and its self-calibration. In Gool, L. V., editor, Pattern Recognition, 24th DAGM Symposium, LNCS, pages 512522. Springer.

Teschner, M., Kimmerle, S., Heidelberger, B., Zachmann, G., Raghupathi, L., A. Fuhrmann, M.-P. C., Faure, F. Magnenat-Thalmann, N., Strasser, W., and Volino, P. (2005). Collision detection for deformable objects. In Computer Graphics Forum.

Wang, X., Kotranza, A., Quarles, J., Lok, B., and Allen, B. D. (2005). Rapidly incorporating real objects for evaluation of engineering designs in a mixed reality environment. In $3 D$ User Interfaces Workshop, IEEE Virtual Reality. 